\title{
A Development of Rapid, Practical and Selective Process for Preparation of $Z$-Oximes
}

\author{
Bo Ram Kim, Gi Hyeon Sung, Jeum-Jong Kim ${ }^{\dagger}$, and Yong-Jin Yoon* \\ Department of Chemistry \& Environmental Biotechnology National Core Research Center, Research Institute of \\ Natural Sciences, Graduate School for Molecular Materials and Nanochemistry, Gyeongsang National University, \\ Jinju Gyeognam 660-701, Korea, *E-mail: yjyoon@gnu.ac.kr \\ ${ }^{\dagger}$ Advanced Solar Technology Research Department, Electronic and Telecommunications Research Institute, \\ Daejeon 305-700, Korea
}

(Received December 28, 2012; Accepted January 22, 2013)

Key words: Oxime, Oximation, Aldehyde, Ketone, Potassium carbonate

Oximes are important functional groups in organic chemistry due to their synthetic utility as protecting groups for carbonyl groups and their ability to form other functionalities, ${ }^{1-4}$ and their biological activity. ${ }^{5}$ Oximes are commonly prepared by condensing aldehydes and ketones with hydroxylamines. These reactions do not always go to completion and reaction times can be long, and therefore there has been interest in more convenient and efficient methods. To avoid the typical disadvantage, conversion of aldehydes and ketones to the corresponding oximes was accomplished by using various catalysts such as organic acid/bases, ${ }^{1,2,6} \mathrm{AcONa}$, alumina, ${ }^{7} \mathrm{TiO}_{2} / \mathrm{SO}_{4}{ }^{2-},{ }^{2}$ silica gel, ${ }^{8}$

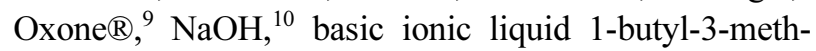
ylimidazolium hydroxide, ${ }^{11}$ polyoxometalates, ${ }^{12} \mathrm{Na}_{2} \mathrm{SO}_{4},{ }^{13}$ and $\mathrm{CuSO} \mathrm{S}_{4} / \mathrm{K}_{2} \mathrm{CO}_{3}{ }^{14}$ under the solvent, the solvent-free or the microwave conditions. These are one and more drowbacks such as long reaction time, use of catalysts, inconvenients due to solid-sate reaction, low yields and limitaion of some carbonyl compounds. On the other hand, $\mathrm{H}$. Sharghi, et al., ${ }^{14}$ reported the catalysis of the stereoselectivity of $\mathrm{CuSO}_{4}$ and $\mathrm{K}_{2} \mathrm{CO}_{3}$ in the oximation of aldehydes and ketones under solvent-free conditions. Although this method show high selectivity, it is inconvenient for the large scale experiments and the industrial process due to the solvent-free condition. Therefore, we attempted to develop a more convenient and efficient solution method. According to the literatures, ${ }^{15}$ treatment of potassium carbonate with methanol generates slightly the potassium methoxide, which may be useful for forming the free $\mathrm{NH}_{2} \mathrm{OH}$ from its salts. We describe the oximation of aldehyde and ketone using $\mathrm{NH}_{2} \mathrm{OH} \cdot \mathrm{HCl} / \mathrm{K}_{2} \mathrm{CO}_{3}$ in methanol solvent.

We selected oximation of acetophenone (1a) with hydrox- ylamine hydrochloride as a model and its behavior was investigated in seven solvents involving methanol (Table 1).

As shown in the Entry 2 in Table 1, compound 1a was treated with hydroxylamine hydrochloride in the presence of potassium carbonate in refluxing 1,4-dioxane to afford the corresponding ketoxime $\mathbf{2 a}$ in excellent yield.

Acetophenone oxime (2a) was also obtained by the use of ethanol or methanol as the solvent at room temperature or at reflux temperature in excellent yields (Entries 9-12 in Table 1), whereas the side reaction was detected for the

Table 1. Screening of the solvents

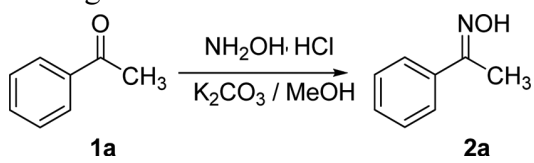

\begin{tabular}{|c|c|c|c|c|}
\hline Entry & Solvent & Conditions & Time (h) & 2a $(\text { Yield } \%)^{a}$ \\
\hline 1 & 1,4-Dioxane & Room temp. & 48 & $-^{b}$ \\
\hline 2 & 1,4-Dioxane & Reflux & 5 & 96 \\
\hline 3 & Diethyl Ether & Room temp. & 48 & $-{ }^{b}$ \\
\hline 4 & Diethyl Ether & Reflux & 48 & 25 \\
\hline 5 & Chloroform & Room temp. & 48 & $-{ }^{b}$ \\
\hline 6 & Chloroform & Reflux & 48 & 13 \\
\hline 7 & Tetrahydrofuran & Room temp. & 48 & $-{ }^{b}$ \\
\hline 8 & Tetrahydrofuran & Reflux & 48 & 30 \\
\hline 9 & Ethanol & Room temp. & 15 & 94 \\
\hline 10 & Ethanol & Reflux & 6 & 92 \\
\hline 11 & Methanol & Room temp. & 8.5 & 98 \\
\hline 12 & Methanol & Reflux & 0.25 & 98 \\
\hline 13 & Water & Room temp. & 48 & trace \\
\hline 14 & Water & Reflux & 4.5 & $60^{c}$ \\
\hline
\end{tabular}

${ }^{a}$ Isolated yield.

${ }^{b}$ Trace.

${ }^{c}$ Hydroxylamine was decomposed in hot water. 
Table 2. Oximation of aldehydes and ketones with hydroxylamine hydrochloride in the presence of potassium carbonate in methanol

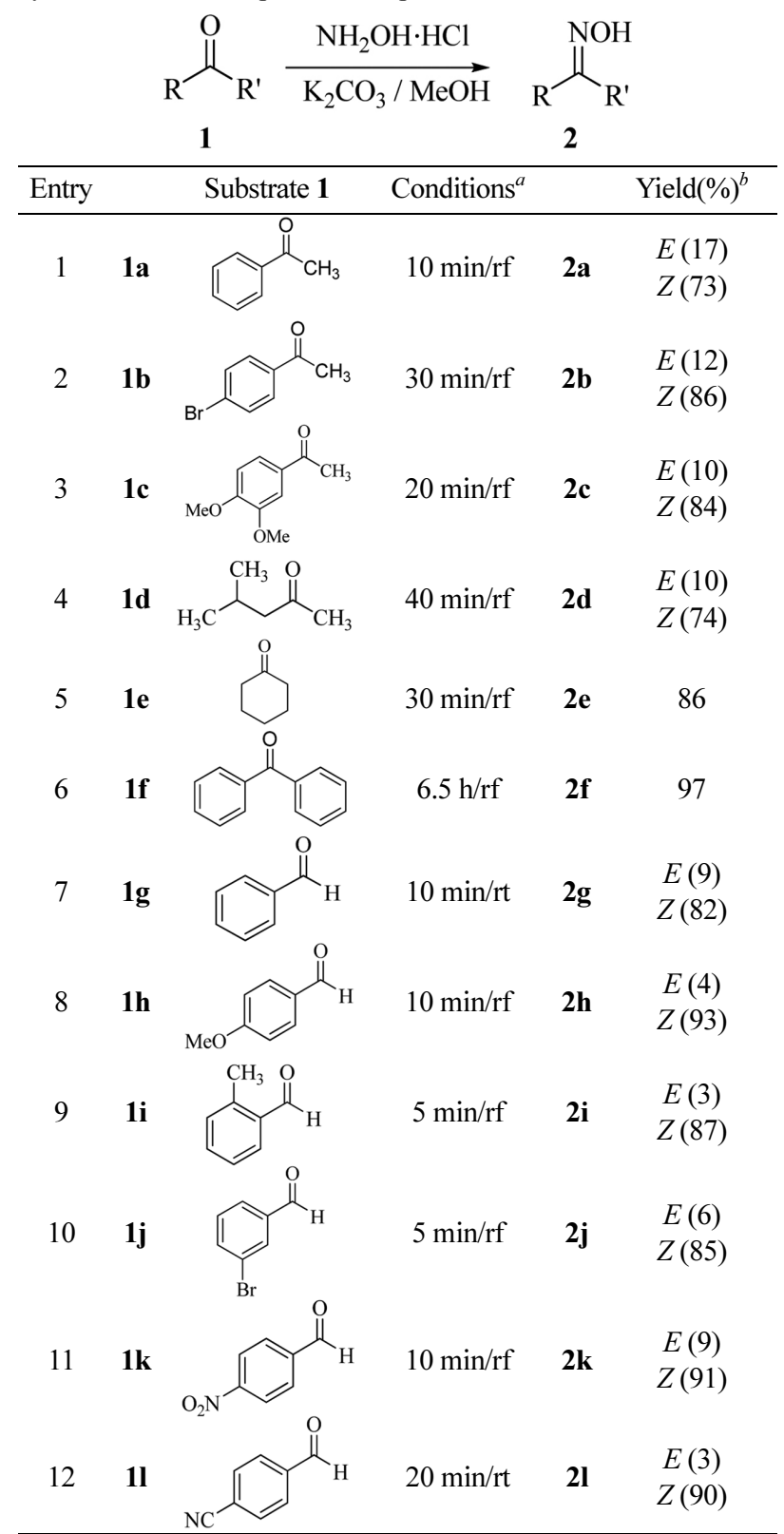

${ }^{a_{\text {rf }}=\text { reflux; } \mathrm{rt}=\text { room temperature. }}$

${ }^{b}$ Isolated yield.

reaction of 1a with hydroxylamine hydrochloride in the presence of potassium carbonate in refluxing water. According to our preliminary results, we selected the methanol as the solvent due to short reaction time and low price.

As shown in Table 2, aliphatic and aromatic ketones 1b1e except for benzophenone (1f) were rapidly and selectively converted to the corresponding $Z$-oximes $\mathbf{2 b}-\mathbf{2} \mathbf{e}$ in good yields. Also, various types of aromatic aldehydes 1g-1l with electron donating and withdrawing groups were rap- idly and selectively converted to the corresponding $Z$ aldoximes $\mathbf{2 g - 2 l}$ in good to excellent yields. In our condition, the oximation of aldehydes is more $Z$-selective than the oximation of ketones. In order to evaluate the utility of industrial process, we examined the one mole scale reaction. Treatment of one mole acetophenone (1a) and benzaldehyde (1g) with hydroxylamine hydrochloride and potassium carbonate in methanol afforded the corresponding oximes $\mathbf{2 a}(E / Z$ ratio $=15: 85,92 \%)$ and $\mathbf{2 g}(E / Z$ ratio $=10: 90,92 \%)$.

The structures of the oximes were established by IR and NMR. In the case of ketoximes, we distinguished two isomers by using the carbon chemical shifts of $\mathrm{C}=\mathrm{NOH}$, that is, the chemical shifts of the $Z$-isomer are detected higher field than the chemical shifts of the $E$-isomer. ${ }^{16}$ In the case of aldoximes, $E / Z$-isomers were distinguished by using the proton chemical shifts of $\mathrm{HC}=\mathrm{N}$ for aldoximes, that is, the proton chemical shifts of the $Z$-isomer are detected lower field than the chemical shifts of the $E$-isomer. ${ }^{17}$

In summury, we have demonstrated the rapid and convenient oximation of ketones and aldehydes with potassium carbonate in methanol at room or reflux temperature in good to excellent yields. Our method has some advantages: use of cheap reagent, mild reaction conditions, high $Z$-selectivity, short reaction time, no side-reactions, good solubility of methanol for carbonyl compounds, the solution reaction and easy work-up.

\section{EXPERIMENTAL}

Melting points were determined with a capillary apparatus and uncorrected. NMR spectra were recorded on a $300 \mathrm{MHz}$ spectrometer with chemical shift values reported in units (ppm) relative to an internal standard (TMS). IR spectra were obtained on SIMADZU FT-IR 8400s spectrophotometer. The open-bed chromatography was carried out on silica gel (70-230 mesh, Merck) using gravity flow. The column was packed with slurries made from the elution solvent.

\section{General Procedure for the Conversion of Aldehydes and Ketones to Oximes}

A mixture of carbonyl compound (1a-1l, $8.3 \mathrm{mmol})$, hydroxylamine hydrochloride $(8.4 \mathrm{mmol}), \mathrm{K}_{2} \mathrm{CO}_{3}(9.1$ $\mathrm{mmol}$ ) and $\mathrm{MeOH}(40 \mathrm{ml})$ was stirred at until the carbonyl compound was disappeared at reflux temperature or at room temperature. After cooling to room temperature, ketoximes $\mathbf{2 a}-\mathbf{2 f}$ were extracted with dichloromethane $(30 \times$ $5 \mathrm{~mL}$ ). The organic layer was separated and dried over 
anhydrous $\mathrm{MgSO}_{4}$. After evaporating the solvent under reduced pressure at below $20{ }^{\circ} \mathrm{C}$, the isomers were then separated from the resulting residue by column chromatography $[n$-hexane : ethyl acetate $=3: 1(\mathrm{v} / \mathrm{v})]$ to give the corresponding $E$ - and $Z$-ketoximes. (Caution: $E$ - ketoxime is evaporating the solvent below $20^{\circ} \mathrm{C}$. And store in freezer).

In the case of aldoximes, the solvent was evaporated under reduced pressure. The resulting residue was washed with cold diethyl ether $(150 \mathrm{~mL})$. The ether solutions were combined, and evaporated under reduced pressure. The isomers were then separated by column chromatography $[n$-hexane : ethyl acetate $=3: 1(\mathrm{v} / \mathrm{v})]$ to give the corresponding $E$ - and $Z$-aldoximes.

\section{(E)-Acetophenone oxime $(\boldsymbol{E}-\mathbf{2 a})$}

Mp 75-76 ${ }^{\circ} \mathrm{C}$ (lit. ${ }^{18} 80-81^{\circ} \mathrm{C}$ ); $\mathrm{R}_{f}=0.23$ (n-hexane : ethyl acetate $=3: 1, \mathrm{v} / \mathrm{v})$; IR (KBr): 3193, 3083, 3053, 3030, 2967, 2920, 2868, 1468, 1436, 1375, 1301, 1267, 1021, 950, 759, 693, $621 \mathrm{~cm}^{-1} ;{ }^{1} \mathrm{H} \mathrm{NMR}\left(300 \mathrm{MHz}, \mathrm{CDCl}_{3}\right): \delta$ $3.31(\mathrm{~s}, 3 \mathrm{H}), 7.36-7.42(\mathrm{~m}, 3 \mathrm{H}), 7.60-7.64(\mathrm{~m}, 2 \mathrm{H}) ;{ }^{13} \mathrm{C}$ $\mathrm{NMR}\left(75 \mathrm{MHz}, \mathrm{CDCl}_{3}\right): \delta 12.37,126.0,128.5,129.3,136.4$, 156.1; HRMS(EI): $m / z$ calcd for $\mathrm{C}_{8} \mathrm{H}_{9} \mathrm{NO}: 135.0684$; found: 135.0684 .

\section{(Z)-Acetophenone oxime $(Z$-2a)}

Mp 52-54 ${ }^{\circ} \mathrm{C}$ (lit. ${ }^{19} 52-55^{\circ} \mathrm{C}$ ); $\mathrm{R}_{f}=0.3$ ( $n$-hexane : ethyl acetate $=3: 1, \mathrm{v} / \mathrm{v})$; IR (KBr): 3292, 3245, 3064, 2926, 1496, 1446, 1370, 1302, 1265, 1079, 1006, 926, 762, 744 $\mathrm{cm}^{-1} ;{ }^{1} \mathrm{H}$ NMR $\left(300 \mathrm{MHz}, \mathrm{CDCl}_{3}\right): \delta 2.30(\mathrm{~s}, 3 \mathrm{H}), 7.36$ $(\mathrm{m}, 3 \mathrm{H}), 7.60-7.62(\mathrm{~m}, 2 \mathrm{H}), 9.86\left(\mathrm{~s}, \mathrm{OH}, \mathrm{D}_{2} \mathrm{O}\right.$ exchangeable ); ${ }^{13} \mathrm{C} \mathrm{NMR}$ (75 MHz, $\mathrm{CDCl}_{3}$ ): $\delta 12.42,126.0,128.5$, 129.2, 136.4, 155.9; HRMS (EI): $m / z$ calcd for $\mathrm{C}_{8} \mathrm{H}_{9} \mathrm{NO}$ : 135.0684; found: 135.0684 .

\section{(E)-4-Bromoacetophenone oxime $(E-2 b)$}

Mp $115-116^{\circ} \mathrm{C} ; \mathrm{R}_{f}=0.2(n$-hexane $:$ ethyl acetate $=3: 1$, v/v); IR (KBr): 3199, 3087, 3054, 2090, 2869, 2838, 1585, 1488, 1460, 1423, 1392, 1264, 1089, 1029, 1010, 942, 820, $748 \mathrm{~cm}^{-1} ;{ }^{1} \mathrm{H} \mathrm{NMR}\left(300 \mathrm{MHz}, \mathrm{CDCl}_{3}\right): \delta 2.27$ (s, 3H), 7.25-7.56 (m, 4H); $\left.{ }^{13} \mathrm{C} \mathrm{NMR} \mathrm{(75} \mathrm{MHz,} \mathrm{CDCl}_{3}\right): \delta$ 12.22, 123.6, 127.6, 131.6, 135.2, 155.2(9); HRMS (EI): $\mathrm{m} / z$ calcd for $\mathrm{C}_{8} \mathrm{H}_{8} \mathrm{NOBr}: 212.9789$; found: 212.9793 .

\section{(Z)-4-Bromoacetophenone oxime $(\boldsymbol{Z}$-2b)}

Mp $124-126^{\circ} \mathrm{C} ; \mathrm{R}_{f}=0.48$ ( $n$-hexane : ethyl acetate $=$ 3:1, v/v); IR (KBr): 3286, 3246, 3082, 3009, 3956, 2914, 1484, 1394, 1309, 1274, 1182, 1069, 1008, 929, 825, 750 $\mathrm{cm}^{-1}$; ${ }^{1} \mathrm{H}$ NMR $\left(300 \mathrm{MHz}, \mathrm{CDCl}_{3}\right.$ ): $\delta 2.27$ (s, 3H), 7.24$7.52(\mathrm{~m}, 4 \mathrm{H}), 8.72\left(\mathrm{~s}, \mathrm{OH} \mathrm{D}_{2} \mathrm{O}\right.$ exchangeable); ${ }^{13} \mathrm{C} \mathrm{NMR}$
(75 MHz, $\left.\mathrm{CDCl}_{3}\right): \delta 12.25,123.6,127.6,131.7,135.2,155.2$ (5); HRMS (EI): $m / z$ calcd for $\mathrm{C}_{8} \mathrm{H}_{8} \mathrm{NOBr}$ : 212.9789; found: 212.9793 .

\section{(E)-3,4-Dimethoxyacetophenone oxime $(E-2 c)$}

Mp 45-47 ${ }^{\circ} \mathrm{C} ; \mathrm{R}_{f}=0.11$ ( $n$-hexane $:$ ethyl acetate $=3: 1$, v/v); IR (KBr): 3249, 3005, 2954, 2921, 2851, 1517, 1463, 1416, 1367, 1274, 1260, 1225, 1175, 1148, 1024, 948, $869,804,764,749 \mathrm{~cm}^{-1}$; ${ }^{1} \mathrm{H}$ NMR (300 MHz, DMSO-d $)$ : $\delta 2.10(\mathrm{~s}, 3 \mathrm{H}), 3.75(\mathrm{~d}, 6 \mathrm{H}, J=9.9 \mathrm{~Hz}), 6.95-7.31(\mathrm{~m}$, $3 \mathrm{H}), 10.59$ (s, OH, $\mathrm{D}_{2} \mathrm{O}$ exchangeable); ${ }^{13} \mathrm{C}$ NMR (75 MHz, DMSO- $\left.d_{6}\right): \delta 21.87,55.39,55.45,110.9,111.6$, 112.3, 121.3, 147.7, 150.1, 158.1; HRMS(EI): $\mathrm{m} / \mathrm{z}$ calcd for $\mathrm{C}_{10} \mathrm{H}_{13} \mathrm{NO}_{3}$ : 195.0895; found: 195.0899.

\section{(Z)-3,4-Dimethoxyacetophenone oxime (Z-2c)}

Mp 141-143 ${ }^{\circ} \mathrm{C} ; \mathrm{R}_{f}=0.17$ ( $n$-hexane $:$ ethyl acetate $=3: 1, \mathrm{v} /$ v); IR (KBr): 3432, 3084, 3011, 2975, 2928, 2843, 1583, $1515,1503,1456,1441,1332,1296,1272,1241,1222$, $1173,1149,1079,1016,998,950,869,812,766 \mathrm{~cm}^{-1} ;{ }^{1} \mathrm{H}$ NMR $\left(300 \mathrm{MHz}, \mathrm{DMSO}-d_{6}\right): \delta 2.14(\mathrm{~s}, 3 \mathrm{H}), 3.77(\mathrm{~s}, 6 \mathrm{H})$, 6.93-6.95 (m, 1H), 7.14-7.17 (m, 1H), 7.28(2)-7.28(4) (m, 1H), 11.03 (s, OH D $2 \mathrm{O}$ exchangeable); ${ }^{13} \mathrm{C}$ NMR (75 MHz, DMSO- $\left.d_{6}\right): \delta 11.33,55.22,55.38,108.2,111.1$, 118.4, 129.5, 148.4, 149.4, 152.3; HRMS(EI): $\mathrm{m} / \mathrm{z}$ calcd for $\mathrm{C}_{10} \mathrm{H}_{13} \mathrm{NO}_{3}$ : 195.0895; found: 195.0899 .

\section{(E)-4-Methylpentan-2-one oxime $(E-2 d)$}

Liquid; $\mathrm{R}_{f}=0.35$ ( $n$-hexane : ethyl acetate $\left.=3: 1, \mathrm{v} / \mathrm{v}\right)$; IR (KBr): 1341, 1265, 1215, 1165, 1108, 1017, 955, 803, $751 \mathrm{~cm}^{-1} ;{ }^{1} \mathrm{H} \mathrm{NMR}\left(300 \mathrm{MHz}, \mathrm{CDCl}_{3}\right): \delta 0.94(\mathrm{~d}, 6 \mathrm{H}, J=$ $6.6 \mathrm{~Hz}), 1.86(0)-1.86(9)(\mathrm{m}, 4 \mathrm{H}), 2.25$ (d, 2H, $J=7.5 \mathrm{~Hz})$, 8.56 (s, OH, $\mathrm{D}_{2} \mathrm{O}$ exchangeable); ${ }^{13} \mathrm{C} \mathrm{NMR}(75 \mathrm{MHz}$, $\mathrm{CDCl}_{3}$ ): $\delta 14.10,25.73,26.90,37.44,158.3$; HRMS(EI): $m / z$ calcd for $\mathrm{C}_{6} \mathrm{H}_{13} \mathrm{NO}$ : 115.0997; found: 115.1008 .

\section{(Z)-4-Methylpentan-2-one oxime ( $Z$-2d)}

Liquid; $\mathrm{R}_{f}=0.55(n$-hexane $:$ ethyl acetate $=3: 1, \mathrm{v} / \mathrm{v})$; IR (KBr): 3422, 2958, 2375, 1629, 1464, 1374, 1112, 974, 931, 875, 832, $736 \mathrm{~cm}^{-1} ;{ }^{1} \mathrm{H}$ NMR (300 MHz, $\left.\mathrm{CDCl}_{3}\right): \delta$ $0.91(\mathrm{~d}, 6 \mathrm{H}, J=6.6 \mathrm{~Hz}), 1.82-1.94(\mathrm{~m}, 4 \mathrm{H}), 2.06(\mathrm{~d}, 2 \mathrm{H}, J$ $=7.2 \mathrm{~Hz}), 9.74\left(\mathrm{~s}, \mathrm{OH}, \mathrm{D}_{2} \mathrm{O}\right.$ exchangeable $) ;{ }^{13} \mathrm{C} \mathrm{NMR}(75$ $\left.\mathrm{MHz}, \mathrm{CDCl}_{3}\right): \delta 13.56,22.36,25.85,44.75,157.9$; HRMS (EI): $m / z$ calcd for $\mathrm{C}_{6} \mathrm{H}_{13} \mathrm{NO}: 115.0997$; found: 115.1008 .

\section{Cyclohexanone oxime (2e)}

Mp 85-87 ${ }^{\circ} \mathrm{C}$ (lit. ${ }^{20} 85-87^{\circ} \mathrm{C}$ ); $\mathrm{R}_{f}=0.5$ ( $n$-hexane : ethyl acetate $=3: 1, \mathrm{v} / \mathrm{v}) ; \mathrm{IR}(\mathrm{KBr}): 3232,3114,2933,2856,2729$, 1600, 1477, 148, 1249, 1222, 991, 958, 896, 763, 655, 
$565,474 \mathrm{~cm}^{-1} ;{ }^{1} \mathrm{H}$ NMR (300 MHz, $\left.\mathrm{CDCl}_{3}\right): \delta 1.56-1.71$ (m, 6H), 2.20-2.25 (m, 2H), 2.49-2.53 (m, 2H), $8.54(\mathrm{~s}$, $1 \mathrm{H}) ;{ }^{13} \mathrm{C}$ NMR (75 MHz, $\mathrm{CDCl}_{3}$ ): $\delta 24.51,25.57,25.77$, 26.86, 32.08, 160.89; HRMS (EI): $m / z$ calcd for $\mathrm{C}_{6} \mathrm{H}_{11} \mathrm{NO}$ : 113.0841; found: 113.0848 .

\section{Benzophenone oxime (2f)}

Mp $136-137^{\circ} \mathrm{C}$ (lit. $\left.{ }^{21} 138-142^{\circ} \mathrm{C}\right) ; \mathrm{R}_{f}=0.45$ ( $n$-hexane $:$ ethyl acetate $=3: 1, \mathrm{v} / \mathrm{v}) ; \mathrm{IR}(\mathrm{KBr}): 3253,3055,2953$, 2923, 2854, 1448, 1327, 1077, 1032, 997, 919, 766, 697 $\mathrm{cm}^{-1}$; ${ }^{1} \mathrm{H}$ NMR (300 MHz, DMSO-d 6 ): $\delta 7.26-7.29$ (m, 2H), 7.37-7.38 (m, 5H), 7.41-7.49 (m, 3H), $11.34(\mathrm{~s}, 1 \mathrm{H}) ;{ }^{13} \mathrm{C}$ NMR (75 MHz, DMSO- $\left.d_{6}\right): \delta 127.4,128.6,128.8(0), 128.8(4)$, 129.3, 133.9, 137.1, 147.3, 155.6; HRMS(EI): $\mathrm{m} / z$ calcd for $\mathrm{C}_{13} \mathrm{H}_{11} \mathrm{NO}$ : 197.0841; found: 197.0844.

\section{(E)-Benzaldehyde oxime (E-2g)}

Mp $113-115^{\circ} \mathrm{C} ; \mathrm{R}_{f}=0.4(n$-hexane $:$ ethyl acetate $=3: 1$, v/v); IR (KBr): 3461, 3156, 2923, 2854, 1639, 1433, 1351, 1268, 954, 850, 754, $691 \mathrm{~cm}^{-1}$; ${ }^{1} \mathrm{H}$ NMR $(300 \mathrm{MHz}$, $\left.\mathrm{CDCl}_{3}\right): \delta 7.36(\mathrm{~s}, 1 \mathrm{H}), 7.39-7.47(\mathrm{~m}, 3 \mathrm{H}), 7.91-7.97(\mathrm{~m}$, $2 \mathrm{H}), 8.35$ (s, OH, $\mathrm{D}_{2} \mathrm{O}$ exchangeable); ${ }^{13} \mathrm{C}$ NMR $(75 \mathrm{MHz}$, $\mathrm{CDCl}_{3}$ ): $\delta 128.4,130.1,130.3,130.8,147.0$; HRMS(EI): $\mathrm{m} / \mathrm{z}$ calcd for $\mathrm{C}_{7} \mathrm{H}_{7} \mathrm{NO}$ : 121.0528; found: 121.0529 .

\section{(Z)-Benzaldehyde oxime (Z-2g)}

Liquid; $\mathrm{R}_{f}=0.57(n$-hexane : ethyl acetate $=3: 1, \mathrm{v} / \mathrm{v})$; IR (KBr): 3388, 2896, 2771, 1954, 1895, 1692, 1634, 1497, 1449, 1292, 1211, 956, 869, $756 \mathrm{~cm}^{-1} ;{ }^{1} \mathrm{H}$ NMR $(300 \mathrm{MHz}$, $\left.\mathrm{CDCl}_{3}\right): \delta 7.32-7.36(\mathrm{~m}, 3 \mathrm{H}), 7.53-7.58(\mathrm{~m}, 2 \mathrm{H}), 8.18(\mathrm{~s}$, 1H), 9.95 (s, OH, $\mathrm{D}_{2} \mathrm{O}$ exchangeable ); ${ }^{13} \mathrm{C}$ NMR $(75 \mathrm{MHz}$, $\mathrm{CDCl}_{3}$ ): $\delta$ 127.0, 128.7, 130.0, 131.6, 150.5; HRMS (EI): $\mathrm{m} / \mathrm{z}$ calcd for $\mathrm{C}_{7} \mathrm{H}_{7} \mathrm{NO}$ : 121.0528; found: 121.0529 .

\section{(E)-4-Methoxybenzaldehyde oxime (E-2h)}

$\mathrm{Mp} 84-85^{\circ} \mathrm{C}\left(\right.$ lit. $\left.{ }^{22} 74^{\circ} \mathrm{C}\right) ; \mathrm{R}_{f}=0.38$ ( $n$-hexane : ethyl acetate $=3: 1, \mathrm{v} / \mathrm{v}) ; \mathrm{IR}(\mathrm{KBr}): 3173,3069,3009,2925,2837$, 2793, 1599, 1506, 1448, 1406, 1351, 1301, 1257, 1172, 1113, $1022,951,827,743 \mathrm{~cm}^{-1} ;{ }^{1} \mathrm{H} N M R\left(300 \mathrm{MHz}, \mathrm{CDCl}_{3}\right): \delta$ $3.83(\mathrm{~s}, 3 \mathrm{H}), 6.90-6.96(\mathrm{~m}, 2 \mathrm{H}), 7.31(\mathrm{~s}, 1 \mathrm{H}), 7.92-7.96(\mathrm{~m}$, 2H), 8.98 (s, OH, $\mathrm{D}_{2} \mathrm{O}$ exchangeable); ${ }^{13} \mathrm{C}$ NMR $(75 \mathrm{MHz}$, $\left.\mathrm{CDCl}_{3}\right): \delta 55.31,113.7,132.0,132.4,146.3,160.6$; HRMS (EI): $m / z$ calcd for $\mathrm{C}_{8} \mathrm{H}_{9} \mathrm{NO}_{2}: 151.0633$; found: 151.0631 .

\section{(Z)-4-Methoxybenzaldehyde oxime (2h)}

Mp 44-46 ${ }^{\circ} \mathrm{C} ; \mathrm{R}_{f}=0.43$ ( $n$-hexane $:$ ethyl acetate $=3: 1$, v/v); IR (KBr): 3301, 3005, 2963, 2937, 2910, 2838, 1607, 1575, 1515, 1463, 1418, 1304, 1252, 1109, 1029, 957, 874, $829 \mathrm{~cm}^{-1} ;{ }^{1} \mathrm{H}$ NMR (300 MHz, $\left.\mathrm{CDCl}_{3}\right): \delta 3.81(\mathrm{~s}, 3 \mathrm{H})$, $6.09(\mathrm{~d}, 2 \mathrm{H}, J=8.76 \mathrm{~Hz}), 7.51(\mathrm{~d}, 2 \mathrm{H}, J=8.7 \mathrm{~Hz}), 8.11$ (s, 1H), 9.03 (s, OH, $\mathrm{D}_{2} \mathrm{O}$ exchangeable ); ${ }^{13} \mathrm{C} \mathrm{NMR}(75 \mathrm{MHz}$, $\left.\mathrm{CDCl}_{3}\right): \delta 55.31,114.2,124.5,128.5,149.8,161.0$; HRMS (EI): $m / z$ calcd for $\mathrm{C}_{8} \mathrm{H}_{9} \mathrm{NO}_{2}$ : 151.0633 ; found: 151.0631 .

\section{(E)-2-Methylbenzaldehyde oxime (E-2i)}

$\mathrm{Mp} 88-89^{\circ} \mathrm{C} ; \mathrm{R}_{f}=0.28$ ( $n$-hexane : ethyl acetate $=3: 1$, v/v); IR (KBr): 3191, 3086, 3030, 2987, 2866, 1656, 1600, 1437, 1328, 1275, 1260, 1217, 1037, 956, 939, 854, 764 $\mathrm{cm}^{-1} ;{ }^{1} \mathrm{H}$ NMR $\left(300 \mathrm{MHz}, \mathrm{CDCl}_{3}\right): \delta 2.39(\mathrm{~s}, 3 \mathrm{H}), 7.22-$ $7.32(\mathrm{~m}, 3 \mathrm{H}), 7.64(\mathrm{~s}, 1 \mathrm{H}), 7.88-7.92(\mathrm{~m}, 1 \mathrm{H}), 8.41$ (s, OH, $\mathrm{D}_{2} \mathrm{O}$ exchangeable); ${ }^{13} \mathrm{C} \mathrm{NMR}\left(75 \mathrm{MHz}, \mathrm{CDCl}_{3}\right): \delta 19.88$, 125.6, 129.2, 129.6, 129.7, 130.4, 136.8, 146.3; HRMS (EI): $\mathrm{m} / z$ calcd for $\mathrm{C}_{8} \mathrm{H}_{9} \mathrm{NO}$ : 135.0684 ; found: 135.0683 .

\section{(Z)-2-Methylbenzaldehyde oxime ( $Z$-2i)}

Mp $46-48^{\circ} \mathrm{C} ; \mathrm{R}_{f}=0.43$ ( $n$-hexane : ethyl acetate $=3: 1$, v/v); IR (KBr): 3317, 3069, 3022, 2984, 2922, 2779, 1622, 1489, 1457, 1433, 1312, 1292, 1226, 1125, 1034, 951, $871,754,716 \mathrm{~cm}^{-1} ;{ }^{1} \mathrm{H}$ NMR $\left(300 \mathrm{MHz}, \mathrm{CDCl}_{3}\right): \delta 2.38$ (s, 3H), 7.14-7.28 (m, 3H), 7.65-7.68 (m, 1H), $8.44(\mathrm{~s}, 1 \mathrm{H})$, 9.72 (s, OH, $\mathrm{D}_{2} \mathrm{O}$ exchangeable). ${ }^{13} \mathrm{C}$ NMR $(75 \mathrm{MHz}$, $\left.\mathrm{CDCl}_{3}\right): \delta 19.77,126.3,126.6,130.0,130.1,130.9,136.2$, 149.3; HRMS (EI): $\mathrm{m} / \mathrm{z}$ calcd for $\mathrm{C}_{8} \mathrm{H}_{9} \mathrm{NO}: 135.0684$; found: 135.0683 .

\section{(E)-3-Bromobenzaldehyde oxime (E-2j)}

Mp $98-100^{\circ} \mathrm{C} ; \mathrm{R}_{f}=0.25(n$-hexane $:$ ethyl acetate $=3: 1$, $\mathrm{v} / \mathrm{v})$; IR (KBr): 3170, 3069, 2840, 2746, 1949, 1685, 1644, 1559, 1463, 1415, 1330, 1269, 1191, 1073, 955, 917, 900, $877,780,749 \mathrm{~cm}^{-1} ;{ }^{1} \mathrm{H} \mathrm{NMR}\left(300 \mathrm{MHz}, \mathrm{CDCl}_{3}\right): \delta 7.31-$ $7.33(\mathrm{~m}, 2 \mathrm{H}), 7.53-7.56(\mathrm{~m}, 1 \mathrm{H}), 7.78-7.81(\mathrm{~m}, 1 \mathrm{H}), 8.15$ $-8.16(\mathrm{~m}, 1 \mathrm{H}) ;{ }^{13} \mathrm{C} \mathrm{NMR}\left(75 \mathrm{MHz}, \mathrm{CDCl}_{3}\right): \delta 129.3,130.0$, 133.1, 133.6, 145.4; HRMS (EI): $m / z$ calcd for $\mathrm{C}_{7} \mathrm{H}_{6} \mathrm{NOBr}$ : 189.9633; found: 189.9635 .

\section{(Z)-3-Bromobenzaldehyde oxime (Z-2j)}

Mp 57-59 ${ }^{\circ} \mathrm{C} ; \mathrm{R}_{f}=0.43$ ( $n$-hexane $:$ ethyl acetate $=3: 1$, v/v); IR (KBr): 3343, 3065, 2939, 2888, 2832, 2752, 1943 , $1695,1566,1475,1421,1351,1310,1272,1200,1105$, 1064, 976, 908, 875, $702 \mathrm{~cm}^{-1}$; ${ }^{1} \mathrm{H}$ NMR $(300 \mathrm{MHz}$, $\left.\mathrm{CDCl}_{3}\right): \delta 7.23-7.28(\mathrm{~m}, 1 \mathrm{H}), 7.47-7.53(\mathrm{~m}, 2 \mathrm{H}), 7.74-$ $7.75(\mathrm{~m}, 1 \mathrm{H}), 8.08(\mathrm{~s}, 1 \mathrm{H}), 8.19$ (s, OH, $\mathrm{D}_{2} \mathrm{O}$ exchangeable); ${ }^{13} \mathrm{C}$ NMR $\left(75 \mathrm{MHz}, \mathrm{CDCl}_{3}\right): \delta 125.6,129.7,130.2,132.9$, 149.0; HRMS(EI): $m / z$ calcd for $\mathrm{C}_{7} \mathrm{H}_{6} \mathrm{NOBr}$ : 189.9633 ; found: 189.9635 .

\section{(E)-4-Nitrobenzaldehyde oxime ( $E$-2k)}

Mp $157-159^{\circ} \mathrm{C}$ (lit. $\left.{ }^{23} 146^{\circ} \mathrm{C}\right) ; \mathrm{R}_{f}=0.31$ ( $n$-hexane : 
ethyl acetate $=3: 1, \mathrm{v} / \mathrm{v}) ; \mathrm{IR}(\mathrm{KBr}): 3180,3067,3014$, 2926, 2880, 2837, 2788, 1601, 1519, 1441, 1401, 1344, 1318, 1264, 1192, 1104, 1013, 967, 942, 909, 852, 840, $746 \mathrm{~cm}^{-1} ;{ }^{1} \mathrm{H}$ NMR (300 MHz, DMSO- $\left.d_{6}\right): \delta 7.67(\mathrm{~s}, 1 \mathrm{H})$, 8.21-8.24 (m, 2H), 8.29-8.32 (m, 2H), 12.27 (s, OH, $\mathrm{D}_{2} \mathrm{O}$ exchangeable); ${ }^{13} \mathrm{C}$ NMR (75 MHz, DMSO- $d_{6}$ ): $\delta 123.6$, 131.3, 136.2, 143.0, 147.0; HRMS (EI): $\mathrm{m} / \mathrm{z}$ calcd for $\mathrm{C}_{7} \mathrm{H}_{6} \mathrm{~N}_{2} \mathrm{O}_{3}$ : 166.0378; found: 166.0378 .

\section{(Z)-4-Nitrobenzaldehyde oxime (Z-2k)}

Mp $128-129^{\circ} \mathrm{C}\left(\mathrm{lit}^{24} 129^{\circ} \mathrm{C}\right) ; \mathrm{R}_{f}=0.44$ ( $n$-hexane : ethyl acetate $=3: 1, \mathrm{v} / \mathrm{v}) ; \operatorname{IR}(\mathrm{KBr}): 3335,3079,2938,1925$, 1803, 1602, 1535, 1347, 1213, 1105, 969, 845, $746 \mathrm{~cm}^{-1}$; ${ }^{1} \mathrm{H}$ NMR $\left(300 \mathrm{MHz}\right.$, DMSO- $\left.d_{6}\right): \delta 7.85-7.89(\mathrm{~m}, 2 \mathrm{H})$, 8.24-8.28 (m, 2H), $8.32(\mathrm{~s}, 1 \mathrm{H}), 11.89$ (s, OH, $\mathrm{D}_{2} \mathrm{O}$ exchangeable); ${ }^{13} \mathrm{C}$ NMR (75 MHz, DMSO- $\left.d_{6}\right): \delta 123.9,127.2$, 139.4, 146.7, 147.4; HRMS (EI): $m / z$ calcd for $\mathrm{C}_{7} \mathrm{H}_{6} \mathrm{~N}_{2} \mathrm{O}_{3}$ : 166.0378; found: 166.0378 .

\section{(E)-4-Cyanobenzaldehyde oxime (E-2l)}

Mp $138-140^{\circ} \mathrm{C} ; \mathrm{R}_{f}=0.1(n$-hexane $:$ ethyl acetate $=3: 1$, v/v); IR (KBr): 3259, 2981, 2963, 2868, 2855, 2227, 1459, 1403, 1275, 1260, 1176, 1082, 916, 828, 763, 749, $704 \mathrm{~cm}^{-1} ;{ }^{1} \mathrm{H}$ NMR $\left(300 \mathrm{MHz}\right.$, DMSO- $\left.d_{6}\right): \delta 7.58(\mathrm{~s}, 1 \mathrm{H})$, 7.74 (d, 2H, $J=8.19 \mathrm{~Hz}), 8.13$ (d, 2H, $J=8.49 \mathrm{~Hz}), 12.15$ (s, OH, $\mathrm{D}_{2} \mathrm{O}$ exchangeable ); ${ }^{13} \mathrm{C}$ NMR (75 MHz, DMSO$\left.d_{6}\right): \delta 111.5,126.9,130.7,132.3,132.6,143.4$; HRMS (EI): $m / z$ calcd for $\mathrm{C}_{8} \mathrm{H}_{6} \mathrm{~N}_{2} \mathrm{O}$ : 146.0480; found: 146.0478 .

\section{(Z)-4-Cyanobenzaldehyde oxime (Z-2I)}

Mp $171-173^{\circ} \mathrm{C} ; \mathrm{R}_{f}=0.2(n$-hexane : ethyl acetate $=3: 1$, v/v); IR (KBr): 3208, 3003, 2980, 2955, 2935, 2896, 2831, 2225, 1399, 1326, 1305, 1278, 1261, 1191, 1101, 1056, 971, $938,873,842,824,750,702 \mathrm{~cm}^{-1}$; ${ }^{1} \mathrm{H}$ NMR $(300 \mathrm{MHz}$, DMSO- $\left.d_{6}\right): \delta 7.78(\mathrm{~d}, 2 \mathrm{H}, J=8.1 \mathrm{~Hz}), 7.87(\mathrm{~d}, 2 \mathrm{H}, J=8.1 \mathrm{~Hz})$, 8.25 (s, 1H), 11.7 (s, OH, $\mathrm{D}_{2} \mathrm{O}$ exchangeable); ${ }^{13} \mathrm{C}$ NMR (75 MHz, DMSO- $\left.d_{6}\right): \delta 111.2,118.6,126.9,132.6,137.5,147.0$; HRMS (EI): $m / z$ calcd for $\mathrm{C}_{8} \mathrm{H}_{6} \mathrm{~N}_{2} \mathrm{O}: 146.0480$; found: 146.0478 .

\section{REFERENCES}

1. Robertson, G. M. Imines and Their N-substituted Derivatives: Oximes and Their O-R Substituted Analogues, in Comprehensive Organic Functional Group Transformations; Katritzky, A. R.; Meth-Cohn, O.; Rees, C. W., Eds.; Elsevier: Oxford, 1995; Vol. 3, p 425.
2. Rossiter, S.; Dobbs, A. P. Imines and Their N-substituted Derivatives: Oximes and Their O-R Substituted Analogues, in Comprehensive Organic Functional Group Transformations; Katritzky, A. R.; Taylor, R. K., Eds.; Elsevier: Oxford, 2005; Vol. 3, p 451.

3. Adams, J. P. J. Chem. Soc., Perkin Trans.1 2000, 125.

4. Abele, E.; Lukevics, E. Org. Prep. Proced. Int. 2000, 32, 235.

5. (a) Yu, F.; Wolin, R. L.; Desai, P. J.; McGovern, P. M.; Dunford, P. J.; Karlsson, L.; Thurmond, R. L. J. Recptor, Ligand and Channel Res. 2010, 3, 37. (b) Kassa, J.; Kuca, K.; Karasova, J.; Musilek, K. Mini-Rev.Med. Chem. 2008, 8, 1134.

6. Osadchenko, I. M.; Tomilov, A. P. Russ. J. Appl. Chem. 2002, 75(3), 511.

7. Touaux, B.; Texier-Boullet, F.; Hamelin, J. Heteroatom Chem. 1998, 9(3), 351.

8. Kiasat, A. R.; Kazemi, F.; Nourbakhsh, K. Phorus, Sulfur Silicon Relat. Elem. 2004, 179, 1193.

9. Xia, J.-J.; Wang, G.-W. Molecules 2007, 12, 231.

10. Damljanoviæ, I.; Vukiæeviæ, M.; Vukiæeviæ, R. D. Monatsh. Chem. 2006, 137, 301.

11. Zang, H.; Wang, M.; Cheng, B.-W.; Song, J. Ultrason. Sonochem. 2009, 16, 301.

12. Sloboda-Rozner, D.; Neumann, R. Green Chem. 2006, 8, 679.

13. Li, J.-T.; Li, X.-L.; Li, T.-S. Ultrason. Sonochem. 2006, 13, 200.

14. Sharghi, H.; Sarvari, M. H. Synlett 2001, 99.

15. (a) Chang, K. T.; Kim, J. J.; Kim, Y. K.; Park, H. Y.; Hyun, B. H.; Shiro, M.; Yoon, Y. J.; Lee, W. S. Heterocycles 2001, 55, 1927. (b) Choi, S. Y.; Shin, S. C.; Yoon, Y. J. J. Heterocycl. Chem. 1991, 28, 385. (c) Kweon, D. H.; Chung, J. W.; Cho, S. D.; Kim, S. K.; Yoon, Y. J. J. Heterocycl. Chem. 1998, 35, 1401.

16. (a) Esteban, J.; Costa, A. M.; Urpi, F.; Vilarrasa, J. Tetrahedron Lett. 2004, 45, 5563. (b) Lichte, R. L.; Roberts, J. D. J. Am. Chem. Soc. 1971, 5218.

17. (a) Lergenuller, M. Klares, U.; Lichtenthaler, F. W. Carbohydr. Res. 2009, 344, 2127. (b) Eshghi, H.; Alizadeh, M. H.; Davamdar, E. J. Korean Chem. Soc. 2008, 52(1), 52.

18. Guillot-Edelheit, G.; Beugelmans, R. Bull. Soc. Chim. France. 1977, 3(Pt. 2), 368.

19. William G. H.; Robert G. B.; Eugene I. I.; Jaime N. D. J. Pharm. Sci. 1977, 66(11), 1602.

20. Eugen. M.; Horst. M. Chem. Ber. 1955, 88, 165.

21. Javad. M.; Mohammad R. N.-J.; Hamideh. H.; Mohammad G. D.; Gerd. K. Chem. Sus. Chem. 2009, 2(3), 248.

22. Brady, O. L.; Mchugh, G. P. J. Chem. Soc. Trans. 1923, 123, 1190.

23. Brady, O. L.; Dudley, R. J. Chem. Soc. Trans. 1923, 123, 2163.

24. Brehm, L. Acta Cryst. 1972, B28, 3646. 